\title{
An approach to studying the neural correlates of reserve
}

\author{
Yaakov Stern $^{1} \mathbb{D}$
}

Published online: 23 July 2016

(C) Springer Science+Business Media New York 2016

\begin{abstract}
The goal of this paper is to review my current understanding of the concepts of cognitive reserve (CR), brain reserve and brain maintenance, and to describe our group's approach to using imaging to study their neural basis. I present a working model for utilizing data regarding brain integrity, clinical status, cognitive activation and CR proxies to develop analyses that can explore the neural basis of cognitive reserve and brain maintenance. The basic model assumes that the effect of brain changes on cognition is mediated by taskrelated activation. We treat $\mathrm{CR}$ as a moderator to understand how task-related activation might vary as a function of CR, or how CR might operate independently of these differences in task-related activation. My hope is that this presentation will spark discussion across groups that study these concepts, allowing us to come to some common agreement on definitions, methodology and approaches.
\end{abstract}

Keywords Cognitive reserve $\cdot$ Brain reserve $\cdot$ Brain maintenance $\cdot$ fMRI

The goal of this paper is to review my current understanding of the concepts of cognitive reserve (CR), brain reserve (BR), and brain maintenance (BM), and to describe our group's approach to using imaging to study their neural basis. Epidemiologic and imaging evidence strongly suggest that CR moderates the clinical impact of age-related brain changes or Alzheimer's disease (AD) pathology. Our working

Yaakov Stern

ys11@columbia.edu

1 Cognitive Neuroscience Division, Department of Neurology, Columbia University College of Physicians and Surgeons, $630 \mathrm{~W}$ 168th St, P\&S Box 16, New York, NY 10032, USA hypothesis is that $\mathrm{CR}$ operates through individual differences in how tasks are processed in the brain. In addition, our work incorporates the concept of $\mathrm{BR}$, in order to account for individual differences in measures of integrity of the brain itself. The concept of BR has taken on greater importance because of increasing evidence that life exposures can reshape the brain and maintain brain integrity, a process called BM.

\section{Working definitions}

The concept of CR refers to differences in cognitive processes that explain differential susceptibility to functional impairment in the presence of pathology. CR can therefore be considered an active model of reserve in that the brain actively attempts to cope with brain damage by using pre-existing cognitive processes or by enlisting compensatory processes (Stern 2002). A set of lifetime experiences as well as other factors may account for differences in CR across individuals.

On the other hand, the concept of BR suggests that structural characteristics of the brain provide resilience against brain aging and pathology: either there is more brain to lose in the face of pathology or atrophy, or the brain substrate supports more elaborate neural networks. Colloquially, BR might be considered the "hardware" while CR the "software." Theoretically, BR encompasses all of the "anatomic" aspects of the brain and can be measured using diverse techniques including imaging, proteomic measures, and advanced neuroscience approaches. Practically, BR might be measured by gray matter volume, cortical thickness or white matter diffusivity.

We had initially characterized BR as a passive form of reserve (Stern 2002) since it does not involve brain networks that actively moderate differential performance at any given level of BR. However, it is now recognized that life 
experiences help maintain brain integrity (for review see Nyberg et al. (2012)). For example increased cognitive activity is associated with preserved whole-brain (Steffener et al. 2016) and hippocampal (Valenzuela et al. 2008) volume. Also, the brain is much more plastic than once imagined; exposures such as exercise are associated with increased total (Colcombe et al. 2006) and hippocampal (Maguire et al. 2000) volume. Interestingly, studies in mouse models suggest increased physical activity may be associated with reduced amyloid load (Adlard et al. 2005). These observations impart new dynamism to BR and its implications for successful aging and reduced incidence of dementia.

Thus, an important accompanying concept is BM. BM refers to the observation that that some people maintain their brains (or their BR) more successfully than do others. Some of the same life exposures associated with differential CR have also been found to be active in BM.

\section{Our research scheme: cross sectional version}

A schematized approach for studying these concepts in crosssectional data, is summarized in Fig. 1a. The figure includes current status of the brain, clinical outcomes including cognitive performance or disease status, and CR. These are the key features needed to characterize $\mathrm{CR}$, since $\mathrm{CR}$ is posited to moderate the relationship between the status of the brain and

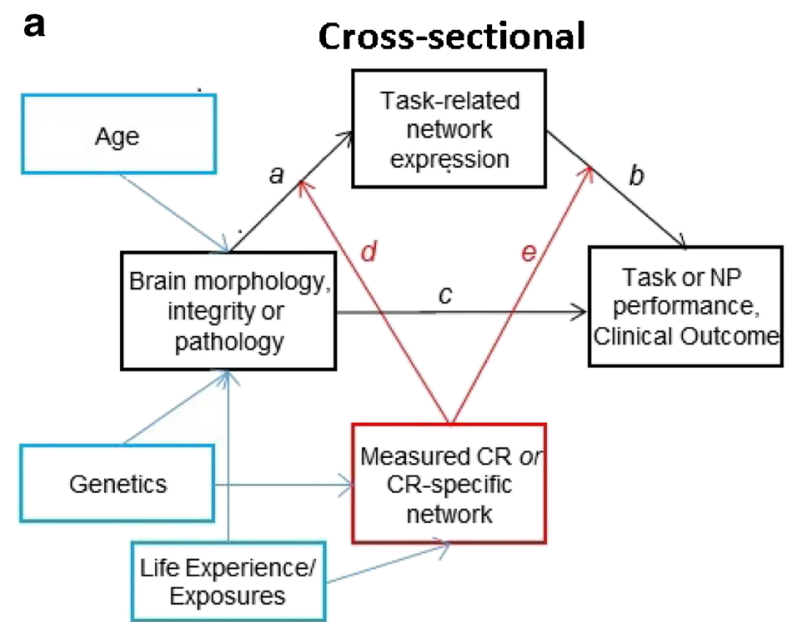

Fig. 1 Cross-sectional (a) and longitudinal (b) models for studying cognitive reserve (CR), brain reserve (BR) and brain maintenance (BM). The models are discussed in-depth in the text. Letters are for identification only and do not have any mathematical significance. Figure 1a assumes that measures of brain morphology, integrity or pathology impact clinical status via path c. CR is represented by the red box; working measures of CR include CR proxies or identified CR brain networks. We consider both the brain measures and $\mathrm{CR}$ to be influenced by age, genetics and life experiences. CR is assumed to moderate the effect of brain status on clinical status, thus producing individual differences in the clinical correlates of a given level of BR and brain pathology. We are attempting to use task-related network expression to better understand the clinical status. The figure also adds a box for task-related activation, which we would like to use to understand the neural implementation of some aspects of CR. The measures used for $\mathrm{CR}$ will be discussed below.

In Fig. 1a, the current status of the brain is characterized by measures of brain morphology and integrity, as well as $\mathrm{AD}$ pathology. Our cross-sectional model incorporates BR but not $\mathrm{BM}$, because $\mathrm{BM}$ cannot be directly observed in crosssectional data. In our studies, we try to incorporate as many imaging modalities as possible to characterize the status of the brain. These include measures of brain volume and cortical thickness, white matter integrity, resting cerebral blood flow and white matter hyperintensity burden. We are acquiring measures of resting BOLD networks, such as those associated with the default mode network. It is an open question whether these networks belong in our characterization of the status of the brain, or should be more accurately considered neural along with fMRI data as components of the neural implementation of CR. We also acquire measures of AD pathology, since this pathology is so common in healthy functioning elderly adults. Thus, one might consider the current status of the brain in the figure to be a representation of BR plus any impinging pathology that is present.

Clinical status is represented by performance on neuropsychological tasks and cognitive tasks used in fMRI studies, as well as by potential clinical diagnoses such as MCI or dementia. Path $\mathrm{c}$ represents the idea that at least a portion of cognitive

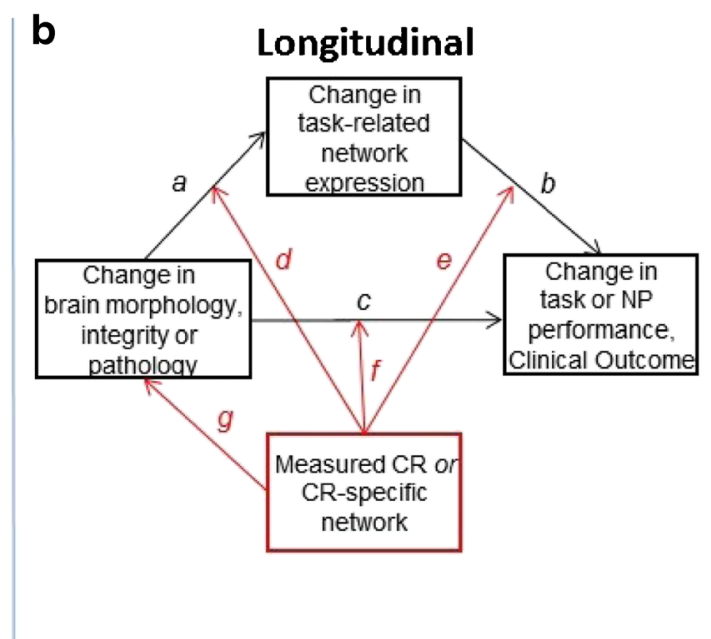

neural implementation of cognitive reserve. We assume that the effect of brain status on clinical status is mediated in part by brain networks captured during task related activation (paths a and b). Path d suggest that $C R$ might moderate between brain status and activation such that given a certain level of brain integrity, some people's task related activation might differ as a function of $\mathrm{CR}$ in informative ways. Path e recognizes that some aspects of CR might not be captured in specific task-related activations, but still might moderate between brain and clinical function. Figure $1 \mathrm{~b}$ extends this model to longitudinal observations. Two key additions are path $\mathrm{f}$, which can assess how $\mathrm{CR}$ moderates the effect of brain change on cognitive change, as well as path g, which addresses the concept of BM 
performance and clinical status can be accounted for by measures of brain morphology and integrity. Implicit in this is that the effect of age on cognition is partially mediated by these brain measures.

Typically, we measure CR via a set of proxies. Our selection of proxy measures for $\mathrm{CR}$ is based on epidemiologic evidence that factor is independently associated with more successful cognitive aging and/or reduced risk of incident dementia. Potential proxies for CR include 1) educational attainment; 2) current or estimated premorbid IQ; 3) measures associated with lifetime occupation; 4) lifetime participation in cognitively stimulating activities; 5) participation in leisure activities, 6) physical activity and exercise, and 7) social engagement. Each proxy can contribute unique information in these models, just as they contribute unique "protection" in epidemiologic models in the form of reduced risk of dementia of slowed cognitive decline with aging. Below, we discuss alternate, more direct measures of $\mathrm{CR}$, including residual measures and generic or task-independent CR networks.

We consider both current BR and CR to be functions of age, genetics and past life exposures. In fact, the same life exposures listed as proxies for CR may also contribute to $\mathrm{BM}$, though probably with differing levels of importance.

One key assumption underlying our investigation of the neural basis of CR is that the relationship of brain integrity to a specific aspect of cognition can be mediated by the underlying cognitive networks that are responsible for performing that function. Thus, we include expression of task-related networks in our model. We can then begin to ask important questions like: "Can the effect of differences in BR on cognitive performance be mediated by differences in the pattern or degree of task-related activation?" This mediation is represented by paths $a$ and $b$.

By definition, we and others (Jones et al. 2011; Bennett et al. 2003) consider CR to moderate the impact of brain changes or pathology on clinical status; i.e. CR affects the relationship between structure and cognition, rather than mediating between them. With two separate brain measures, gray matter volume ROIs (Steffener et al. 2014a) and a covariance measure summarizing fractional anisotropy (FA) in white matter tracts (Gazes et al. 2016), we found that a CR proxy moderated the relationship between brain and cognition such that given a certain gray matter volume or diffusivity status, individuals with higher $\mathrm{CR}$ performed better on cognitive tasks. This suggests that individuals with higher CR are making "better use" of the neural resources available, consistent with the idea of neural reserve as described below. With fMRI analyses, we can investigate how this CR moderation might result in differential brain activity; these individual variations in network activity may in turn mediate the effect of brain change on cognition. Our use of CR proxies as moderators in our analytic models thus allows us to identify these brain network differences.
In Fig. 1a, we posit that $\mathrm{CR}$ could operate as a moderator that supports task-related network expression in the presence of brain changes or pathology (path d). As discussed below, the moderating effect of CR could be reflected in more resilient or flexible cognitive networks. For example, given the same degree of focal volume loss in a network important for working memory, we have noted that individuals with higher IQ have higher network efficiency (Stern et al. 2003). The great majority of our studies of the neural implementation of CR have used this approach. However, even if brain changes do impair specific task-related networks, CR could moderate the effect of these changes on performance or clinical outcomes in a manner that is independent of task-related activation. This moderation might be reflected by the presence of task-invariant networks whose expression is correlated with CR. This possibility is reflected in path e. Implementation of this cross-sectional model is discussed in more detail below. Subsequently, we discuss how the model can be extended to incorporate longitudinal data.

\section{Exploration of the neural implementations of cognitive reserve}

CR implies that some aspects of genetics, experience and lifestyle impart differential susceptibility to underlying age- and disease-related brain changes. CR can be defined as the resilience or plasticity of cognitive networks in the face of disruption. To yield insights into the neural implementation of CR, we can use task-based fMRI studies to characterize how the functioning brain adapts to these physical brain changes in order to maintain cognitive performance. I have suggested several ways in which CR can be neurally implemented (Stern 2009).

Neural reserve The concept of neural reserve posits that interindividual variability in the brain networks that underlie task performance of the healthy brain might cause some people to be more capable than others of coping with the demands imposed by brain changes or pathology. In research applications, some, but not all, aspects of neural reserve would be observed in the box labeled "task related network expression" in Fig. 1a.

One facet of neural reserve that has been systematically explored is differential network efficiency and capacity. In this context, a more efficient network will show less activation in order to produce the same (or higher) level of performance. We realize that other, more computational, definitions of efficiency have been used, but refer here to this simpler concept of efficiency. Capacity can be characterized by the maximal ability to activate a network as cognitive tasks become very demanding. We have demonstrated that a person with higher proxy measures of $\mathrm{CR}$, such as higher IQ, shows patterns of 
task-related activation that are more efficient and have higher capacity (Habeck et al. 2003, 2005; Stern et al. 2003). We have also demonstrated that given a certain degree of brain change, e.g. volume loss, individuals with higher CR can maintain greater network efficiency (Steffener et al. 2009).

Neural reserve also incorporates the idea of greater network flexibility. Some individuals may have more flexible or varied approaches to any particular cognitive activity. This again could result in being better able to withstand brain changes or pathology. Network flexibility is more difficult to ascertain with imaging approaches since it requires the ability to detect individual differences in the utilization of the at least two different networks that underlie flexibility. Without a priori knowledge of these networks, this variability typically could not be detected. We have been using cognitive experimental approaches, such as paradigms that test flexibility in problem solving, to explore this aspect of CR (Barulli et al. 2013).

In pursuing the neural instantiation of neural reserve with fMRI studies, we have often reported age-related differential increases in activation with increasing task load, consistent with efficiency/capacity differences (Stern et al. 2012), and have explored networks that were differentially sensitive to increasing task demand used during working memory tasks (Blumen et al. 2011). We have thoroughly modeled the impact of brain volume changes and the moderating effect of CR using our Letter Sternberg (LS) task (Steffener et al. 2009, 2011; Steffener and Stern 2012). Similarly, using an executive task we have noted age-related losses in network efficiency (Gazes et al. 2012, 2015) that mediate between age and task performance (Steffener et al. 2014b); the efficiency of these networks is influenced by brain volume (Steffener et al. 2016).

Neural compensation We define neural compensation as task-related activation that occurs only in the presence of structural brain changes; i.e. this may result in improved performance for those who compensate (e.g. HAROLD (Cabeza 2002; Cabeza et al. 2002)). However, we have noted that a compensatory network is often used when the primary network has lost function (Zarahn et al. 2007; Steffener et al. 2009), as have others (Grady et al. 1994; Reuter-Lorenz 2002; Madden et al. 1999). Again, Fig. 1a incorporates neural compensation into the box labeled "task-related network expression."

Task-invariant networks Our study of neural reserve and neural compensation rely mainly on task-specific fMRI activation data, and are therefore relevant to path $d$ in Fig. 1a. However, we have demonstrated that generic, "task-invariant networks" may also be elicited during the performance of disparate tasks (Stern et al. 2008). By subserving some general as opposed to task-specific function, these task-invariant networks might allow someone to cope with both pathology and task-related network changes in order to maintain effective functioning. Thus these task-invariant networks are expressed as a function of $\mathrm{CR}$, and also may allow people to maintain function in the face of age- or disease-related brain changes. This concept of task-invariant networks is relevant to path e in Fig. 1a, where CR moderates the total effect of brain and taskrelated neural network changes in order to maintain performance.

Formally, these task-invariant networks may be viewed as another manifestation of neural reserve, in that they are most likely present in healthy individuals and influenced by life exposures. Identifying such networks can also help to begin addressing the question of whether CR is simply the aggregation of specific task-related skills, or if $\mathrm{CR}$ can operate in a more generic, task invariant fashion.

In one study, we attempted to identify a task-invariant CR network by extracting an fMRI covariance pattern that was expressed across two different, unrelated tasks, and whose degree of expression correlated with standard proxies of CR (Stern et al. 2008). With increasing CR there was increased expression in right and left superior frontal gyrus and concomitant reduced expression in left medial frontal gyrus. Such a network was noted in young but not older participants. We are currently attempting to apply this approach to our current studies.

\section{Longitudinal studies}

The promise of better understanding CR and BR is that they have strong implications for preservation of function over time. Thus, the neural mechanisms underlying reserve are optimally studied in a longitudinal context. It is now wellrecognized that prospective data is required in order to evaluate how the effect of age on cognition may be mediated by brain changes (Lindenberger et al. 2011; Maxwell and Cole 2007). The same theoretical and analytic issues are even more acute when exploring how BR and CR may influence the progression of cognitive aging and emergence of dementia. Longitudinal studies allow us to directly evaluate whether and how baseline measures of BR and CR result in preserved brain structure and/or cognitive function. More importantly, the theories of reserve rest on the idea that some aspects of lifestyle and behavior moderate the negative effects of advancing age; therefore, the greatest evidence for the presence and mechanisms of reserve comes from longitudinal follow-up. Longitudinal analyses allow us to study reserve in action, i.e. in the presence of observed, progressing brain changes. Longitudinal studies can therefore help establish causal links that heretofore have been merely theorized to exist between brain change, $\mathrm{CR}$ networks/proxies and more successful cognitive or brain aging.

Figure $1 \mathrm{~b}$ addresses how prospectively collected data can allow us to incorporate change over time in all of our measures 
and better address models of $\mathrm{CR}, \mathrm{BR}$ and $\mathrm{BM}$. We can directly explore how changes in brain morphology and integrity impact cognitive and functional status, and are moderated by CR (Path f). Longitudinal data also provide a more appropriate venue for addressing paths $d$ and e. In path $\mathrm{d}, \mathrm{CR}$ moderates the relationship between changes in brain subsequent task-related activation. In path e, CR might be associated with task-invariant networks that moderate between altered brain and task-related activation on one hand and performance on the other. In addition, because we are now measuring change in BR, longitudinal data allows us to directly assess the determinants of BM (path g) using many of the same life exposure measures that are applied to CR. Although for brevity we did not repeat the inclusion of age, genetics and life exposures in Fig. 1b, we hypothesize that these factors influence the rate of change in BM or pathology as well as CR.

Recently, there has been intense interest in the "preclinical" phase of $\mathrm{AD}$, where the underlying pathologic changes are present, but cognitive changes are subtle or not observed (Sperling et al. 2011). It is now well-recognized that CR is very important in understanding this phase. For example, a more recent version of the well-known Jack et al. (2013) hypothetical model of dynamic biomarkers underlying the development of $\mathrm{AD}$ now recognizes that there is inter-individual variability in the cognitive changes associated with any level of pathology (see Jack et al. (2013), Figure 6). There have also been suggestions that $\mathrm{CR}$ proxies might directly impact the development of AD pathology (e.g. (eg., Landau et al. 2012; Adlard et al. 2005). Prospective data are crucial to address these issues.

\section{Directly measuring CR}

We have been systematically examining and developing models of BM and CR. We replicated the residual approach proposed by Reed et al. (2010) for measuring CR in our epidemiologic data (Zahodne et al. 2013). The variance in memory performance accounted for $\mathrm{r}$ by brain measures and by demographics was quantified. The remaining, residual variance behaved like a direct measure of CR: it correlated with $\mathrm{CR}$ proxies and predicted subsequent cognitive decline and dementia. We also examined changes in this residual estimate of CR over time (Zahodne et al. 2015). We have now developed a parallel approach to measuring BM in the data from the current study. We first used a weighted combination of gray matter regional volumes to predict chronological age and defined the difference between actual and chronological age as a measure of BM. Several life exposures, including education, estimated IQ, and physical activity, were associated with increased BM (Steffener et al. 2016). In a subsequent set of analyses (Habeck et al. (2016)) we extended this approach by incorporating multiple measures of brain integrity to estimate age and then BM. In this study, also derived residual measures for $\mathrm{CR}$ using multiple brain integrity predictors. Interestingly, we found that the $\mathrm{CR}$ residual derived from four different cognitive abilities all tapped into the same underlying process.

Eventually, we hope to identify task-invariant networks whose expression is a direct measure of CR. One potential approach for identifying such networks was discussed above, where we sought a network that was expressed in two disparate tasks and whose expression correlated with CR. We are currently working an extension of this approach, by trying to identify a single network expressed during the performance of many different tasks and whose expression correlates with CR proxies. Such a network can then be tested by forward applying it to data from other tasks. If it is truly a task invariant CR network, then its expression in this forward application should be correlated with CR. Similarly, in Fig. 1 we could replace CR proxies with expression of a putative task-invariant network. We could then test whether network expression provides the same moderation that can be seen using $\mathrm{CR}$ proxies in both paths $\mathrm{d}$ and $\mathrm{e}$. In other words, the taskinvariant network should act in a manner that is consonant with CR.

\section{Conclusion}

In this paper I have tried to define our understanding of the concepts of CR, BR, and BM. I have also attempted to explicate the models and approaches that our group is using to explore these concepts. My hope is that this presentation will spark discussion across groups that study these concepts, allowing us to come to some common agreement on definitions, methodology and approaches.

Acknowledgments This work was supported by a grant from the National Institute on Aging (RO1 AG26158).

\section{Compliance with ethical standards}

Funding This study was funded by National Institute on Aging (RO1 AG26158).

Conflict of interest Dr. Stern declares that he has no conflicts of interest.

Ethical approval All procedures performed in studies involving human participants were in accordance with the ethical standards of the institutional and/or national research committee and with the 1964 Helsinki declaration and its later amendments or comparable ethical standards.

Informed consent Informed consent was obtained from all individual participants included in the study. 


\section{References}

Adlard, P. A., Perreau, V. M., Pop, V., \& Cotman, C. W. (2005). Voluntary exercise decreases amyloid load in a transgenic model of Alzheimer's disease. The Journal of Neuroscience, 25(17), 42174221. doi:10.1523/JNEUROSCI.0496-05.2005.

Barulli, D. J., Rakitin, B. C., Lemaire, P., \& Stern, Y. (2013). The influence of cognitive reserve on strategy selection in normal aging. Journal of the International Neuropsychological Society, 19, 1-4.

Bennett, D. A., Wilson, R. S., Schneider, J. A., Evans, D. A., Mendes De Leon, C. F., Arnold, S. E., et al. (2003). Education modifies the relation of $\mathrm{AD}$ pathology to level of cognitive function in older persons. Neurology, 60(12), 1909-1915.

Blumen, H. M., Gazes, Y., Habeck, C., Kumar, A., Steffener, J., Rakitin, B. C., et al. (2011). Neural networks associated with the speedaccuracy tradeoff: evidence from the response signal method. Behavioural Brain Research, 224(2), 397-402. doi:10.1016/j. bbr.2011.06.004.

Cabeza, R. (2002). Hemispheric asymmetry reduction in older adults: the HAROLD model. Psychology and Aging, 17(1), 85-100.

Cabeza, R., Anderson, N. D., Locantore, J. K., \& McIntosh, A. R. (2002). Aging gracefully: compensatory brain activity in high-performing older adults. NeuroImage, 17(3), 1394-1402.

Colcombe, S. J., Erickson, K. I., Scalf, P. E., Kim, J. S., Prakash, R., McAuley, E., et al. (2006). Aerobic exercise training increases brain volume in aging humans. The Journals of Gerontology. Series A, Biological Sciences and Medical Sciences, 61(11), 1166-1170.

Gazes, Y., Rakitin, B. C., Habeck, C., Steffener, J., \& Stern, Y. (2012). Age differences of multivariate network expressions during taskswitching and their associations with behavior. Neuropsychologia, 50(14), 3509-3518. doi:10.1016/j.neuropsychologia.2012.09.039.

Gazes, Y., Habeck, C., O'Shea, D., Razlighi, Q. R., Steffener, J., \& Stern, Y. (2015). Functional network mediates age-related differences in reaction time: a replication and extension study. Brain and Behavior, 5(5), e00324. doi:10.1002/brb3.324.

Gazes, Y., Bowman, F. D., Razlighi, Q. R., O'Shea, D., Stern, Y., \& Habeck, C. (2016). White matter tract covariance patterns predict age-declining cognitive abilities. NeuroImage, 125, 53-60. doi:10.1016/j.neuroimage.2015.10.016.

Grady, C. L., Maisog, J. M., Horwitz, B., Ungerleider, L. G., Mentis, M. J., Salerno, J. A., et al. (1994). Age-related changes in cortical blood flow activation during visual processing of faces and location. The Journal of Neuroscience, 14(3 Pt 2), 1450-1462.

Habeck, C., Hilton, H. J., Zarahn, E., Flynn, J., Moeller, J., \& Stern, Y. (2003). Relation of cognitive reserve and task performance to expression of regional covariance networks in an event-related fMRI study of nonverbal memory. NeuroImage, 20(3), 1723-1733.

Habeck, C., Rakitin, B. C., Moeller, J., Scarmeas, N., Zarahn, E., Brown, T., et al. (2005). An event-related fMRI study of the neural networks underlying the encoding, maintenance, and retrieval phase in a delayed-match-to-sample task. Brain Research. Cognitive Brain Research, 23(2-3), 207-220.

Habeck, C., Steffener, J., Gazes, Y., \& Stern, Y. (2016). Cognitive reserve and brain maintenance: two orthogonal concepts. Cerebral Cortex (in press).

Jack Jr., C. R., Knopman, D. S., Jagust, W. J., Petersen, R. C., Weiner, M. W., Aisen, P. S., et al. (2013). Tracking pathophysiological processes in Alzheimer's disease: an updated hypothetical model of dynamic biomarkers. Lancet Neurology, 12(2), 207-216. doi:10.1016/S14744422(12)70291-0.

Jones, R. N., Manly, J., Glymour, M. M., Rentz, D. M., Jefferson, A. L., \& Stern, Y. (2011). Conceptual and measurement challenges in research on cognitive reserve. Journal of the International Neuropsychological Society, 17(4), 593-601. doi:10.1017 /S1355617710001748.
Landau, S. M., Marks, S. M., Mormino, E. C., Rabinovici, G. D., Oh, H., O'Neil, J. P., et al. (2012). Association of lifetime cognitive engagement and low beta-amyloid deposition. Archives of Neurology, 69(5), 623-629. doi:10.1001/archneurol.2011.2748.

Lindenberger, U., von Oertzen, T., Ghisletta, P., \& Hertzog, C. (2011). Cross-sectional age variance extraction: what's change got to do with it? Psychology and Aging, 26(1), 34-47. doi:10.1037 /a0020525.

Madden, D. J., Turkington, T. G., Provenzale, J. M., Denny, L. L., Hawk, T. C., Gottlob, L. R., et al. (1999). Adult age differences in the functional neuroanatomy of verbal recognition memory. Human Brain Mapping, 7(2), 115-135.

Maguire, E. A., Gadian, D. G., Johnsrude, I. S., Good, C. D., Ashburner, J., Frackowiak, R. S., et al. (2000). Navigation-related structural change in the hippocampi of taxi drivers. Proceedings of the National Academy of Sciences of the United States of America, 97(8), 4398-4403.

Maxwell, S. E., \& Cole, D. A. (2007). Bias in cross-sectional analyses of longitudinal mediation. Psychological Methods, 12(1), 23-44. doi:10.1037/1082-989X.12.1.23.

Nyberg, L., Lovden, M., Riklund, K., Lindenberger, U., \& Backman, L. (2012). Memory aging and brain maintenance. Trends in Cognitive Sciences, 16(5), 292-305. doi:10.1016/j.tics.2012.04.005.

Reed, B. R., Mungas, D., Farias, S. T., Harvey, D., Beckett, L., Widaman, K., et al. (2010). Measuring cognitive reserve based on the decomposition of episodic memory variance. Brain, 133(Pt 8), 2196-2209. doi:10.1093/brain/awq154.

Reuter-Lorenz, P. (2002). New visions of the aging mind and brain. Trends in Cognitive Sciences, 6(9), 394.

Sperling, R. A., Aisen, P. S., Beckett, L. A., Bennett, D. A., Craft, S., Fagan, A. M., et al. (2011). Toward defining the preclinical stages of Alzheimer's disease: recommendations from the National Institute on Aging-Alzheimer's Association workgroups on diagnostic guidelines for Alzheimer's disease. Alzheimers Dement, 7(3), 280292. doi:10.1016/j.jalz.2011.03.003.

Steffener, J., \& Stern, Y. (2012). Exploring the neural basis of cognitive reserve in aging. Biochimica et Biophysica Acta, 1822(3), 467-473. doi:10.1016/j.bbadis.2011.09.012.

Steffener, J., Brickman, A. M., Rakitin, B. C., Gazes, Y., \& Stern, Y. (2009). The impact of age-related changes on working memory functional activity. Brain Imaging and Behavior, 3(2), 142-153. doi:10.1007/s11682-008-9056-X.

Steffener, J., Reuben, A., Rakitin, B. C., \& Stern, Y. (2011). Supporting performance in the face of age-related neural changes: testing mechanistic roles of cognitive reserve. Brain Imaging and Behavior, 22(4), 655-669. doi:10.1007/s11682-011-9125-4.

Steffener, J., Barulli, D., Habeck, C., O'Shea, D., Razlighi, Q., \& Stern, Y. (2014a). The role of education and verbal abilities in altering the effect of age-related gray matter differences on cognition. PloS One, 9(3), e91196. doi:10.1371/journal.pone.0091196.

Steffener, J., Barulli, D., Habeck, C., \& Stern, Y. (2014b). Neuroimaging explanations of age-related differences in task performance. Frontiers in Aging Neuroscience, 6, 46. doi:10.3389/fnagi.2014.00046.

Steffener, J., Habeck, C., O'Shea, D., Razlighi, Q., Bherer, L., \& Stern, Y. (2016). Differences between chronological and brain age are related to education and self-reported physical activity. Neurobiology of Aging, 40, 138-144. doi:10.1016/j.neurobiolaging.2016.01.014.

Stern, Y. (2002). What is cognitive reserve? Theory and research application of the reserve concept. Journal of the International Neuropsychological Society, 8, 448-460.

Stern, Y. (2009). Cognitive reserve. Neuropsychologia, 47(10), 20152028. doi:10.1016/j.neuropsychologia.2009.03.004.

Stern, Y., Zarahn, E., Hilton, H. J., Delapaz, R., Flynn, J., \& Rakitin, B. (2003). Exploring the neural basis of cognitive reserve. Journal of Clinical and Experimental Neuropsychology, 5, 691-701. 
Stern, Y., Zarahn, E., Habeck, C., Holtzer, R., Rakitin, B. C., Kumar, A., et al. (2008). A common neural network for cognitive reserve in verbal and object working memory in young but not old. Cerebral Cortex, 18(4), 959-967. doi:10.1093/cercor/bhm134.

Stern, Y., Rakitin, B. C., Habeck, C., Gazes, Y., Steffener, J., Kumar, A., et al. (2012). Task difficulty modulates young-old differences in network expression. Brain Research, 1435, 130-145. doi:10.1016 /j.brainres.2011.11.061.

Valenzuela, M. J., Sachdev, P., Wen, W., Chen, X., \& Brodaty, H. (2008). Lifespan mental activity predicts diminished rate of hippocampal atrophy. PloS One, 3(7), e2598. doi:10.1371/journal.pone.0002598.

Zahodne, L. B., Manly, J. J., Brickman, A. M., Siedlecki, K. L., Decarli, C., \& Stern, Y. (2013). Quantifying cognitive reserve in older adults by decomposing episodic memory variance: replication and extension. Journal of the International Neuropsychological Society, 19(8), 854-862. doi:10.1017/S1355617713000738.

Zahodne, L. B., Manly, J. J., Brickman, A. M., Narkhede, A., Griffith, E. Y., Guzman, V. A., et al. (2015). Is residual memory variance a valid method for quantifying cognitive reserve? A longitudinal application. Neuropsychologia, 77, 260-266. doi:10.1016/j. neuropsychologia.2015.09.009.

Zarahn, E., Rakitin, B., Abela, D., Flynn, J., \& Stern, Y. (2007). Agerelated changes in brain activation during a delayed item recognition task. Neurobiology of Aging, 28(5), 784-798. doi:10.1016/j. neurobiolaging.2006.03.002. 